\title{
Rural Indonesia women's traditional beliefs about antenatal care
}

Yenita Agus ${ }^{1,3+}$, Shigeko Horiuchi ${ }^{1,4^{*}+}$ and Sarah E Porter ${ }^{1,2}$

\begin{abstract}
Background: The Indonesia Maternal Mortality Rate (MMR) of 420/100.00 live births remains among the highest in East Asia while coverage of births assisted by skilled providers is still low. Traditional beliefs have been a key factor associated with the choice between midwives or traditional birth attendants (TBA) and the low number of antenatal care visits in rural West Sumatra.

Methods: We conducted three focus groups with 16 women from rural West Java to describe their perception regarding issues related to traditional beliefs. Focus group discussions provided data for the content analysis.

Results: The majority of the 16 women interviewed was from Village Dago, West Java and had only an elementary school education. Their ages ranged from 19 to 40 years. Most were multiparous housewives with an income of IDR 918.750 per month, which was lower than the monthly income in West Java (IDR. 1.172.060). Emerging from the focus group discussion were four main themes regarding their pregnancy and traditional beliefs: 1) pregnancy was a normal cycle in women's life (pregnancy is a natural phenomena, not a sickness; no recognition of danger signs during pregnancy and death of baby or mother during pregnancy was brought about by God's will); 2) women followed the traditional beliefs (positive motivation to follow the traditional beliefs and fear of not following the traditional beliefs); 3) relying on TBA called paraji rather than midwife (parajis are kind, tolerant and patient and have more experience than midwives; more accessibility than midwives and encouragement of natural birth) and 4) midwives are more secure than paraji; (they use a medical standard of care).

Conclusions: Women's beliefs grounded in religion and tradition permeated the village culture making it difficult to counter their long held health practices with practices based on recent advances in health care. Use of TBA in this village was still dominant and women believed that following traditional beliefs led to a healthy pregnancy therefore, they also followed all relatives' suggestions. Understanding the complexities of local culture is the first step to improving women's awareness of how to preserve their pregnancy and prevent complications.
\end{abstract}

Keywords: Pregnancy complications, Rural women, Traditional beliefs, Traditional births attendant

\section{Background}

Improving maternal health is one of the eight Millennium Development Goals adopted by the international health community in 2000 [1]. The Indonesia Maternal Mortality Rate (MMR) remains among the highest in East Asia with 420 deaths per 100,000 live births while, 10,000 women continue to die of childbirth-related causes every year [2,3]. The WHO

\footnotetext{
* Correspondence: shigeko-horiuchi@sın.ac.jp

${ }^{\dagger}$ Equal contributors

'St. Luke's College of Nursing, 10-1 Akashi-cho Chuo-ku, Tokyo 104-0044, Japan

${ }^{4}$ St. Luke's Birth Clinic, 1-24 Akashi- cho, Chuo-ku, Tokyo 104-0044, Japan

Full list of author information is available at the end of the article
}

report [2] noted that coverage of births assisted by skilled providers is still low especially in rural areas, where traditional births attendants (TBAs) assisted more than $50 \%$ of deliveries. In other words TBAs who have minimal training attend the majority of births [4].

The high cost of medical expenses, poor quality of emergency obstetric care and an inadequate number of health specialists to manage complications continue to discourage women from seeking care when needed. In addition, shortage and maldistribution of health providers are problems requiring attention [3,4]. Despite the lack of qualified health care providers, a previous study

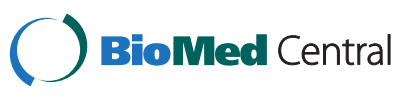


[5] found that traditional beliefs were the greatest contributing factor influencing the preference for TBAs. It was also identified that some of the women especially in rural areas agreed that following the traditional belief was necessary. The UNFPA report found that millions of women were affected by cultural traditions that had implications for their well-being, including the denial of modern medicine during childbirth [6].

Culture is how individuals learn the values, beliefs, norms and way of life that influence their thinking, decisions and actions in certain ways [7]. It is presumed that this discrimination has been woven into the traditional cultural. Family as a unit of culture is therefore an influencing factor for women in following the traditional beliefs and also encouraging women to seeking care during pregnancy $[5,8]$. For example, most women in Bali believed that trusting family advice is necessary [9].

Women's preference for TBAs was more that just a tradition, because TBA's provided the patient with kindness and caring; they had skills plus respect for the local customs which were highly valued $[10,11]$. The TBA understood the local customs. The health care professionals, usually coming from or being educated from a different perspective often failed to understand the significance of the traditional practices and had little knowledge about the traditional beliefs [12].

\section{Statement of the problem}

While there are multiple interacting factors affecting birth outcomes in Indonesia there remain areas where women could chose to access trained midwives as opposed to the TBA. However, many women especially in rural areas still prefer to go to TBAs for assistance during pregnancy. They believe that TBA services are part of the traditional beliefs during pregnancy. This situation is predicted as one of the factors contributing the low number of ANC visit in Indonesia.

In-depth understanding of antenatal care in relationship to traditional beliefs requires investigation using in depth interviews. Health care providers need to know the traditional beliefs of the women, families and communities for whom they provide care; through women's own discussion about their health problems and ANC services their perceptions will become more easily understood. This study focuses on the factors regarding preferences for TBAs or midwives and the role that traditional beliefs might have in their decision.

\section{Purpose}

The aim of the study was to describe women's perception regarding issues related to traditional beliefs during their pregnancy in rural area of Indonesia. The study explored women's perception regarding traditional beliefs, including their reasons for using or not using
ANC services during their pregnancy. Women's perceptions were interpreted within the context of traditional beliefs related to views about pregnancy and health care.

\section{Methods \\ Design}

This study used a qualitative exploratory cross-section design because little was known about women's perceptions of antenatal care and traditional beliefs.

\section{Setting and sample Characteristics of the setting}

The data were collected in the rural area near Bogor in West Java, a province of Indonesia. The capital of the province is Bandung. According to the Indonesian Demographic Health Survey [13] 18\% Indonesia women graduated from secondary school whereas $22 \%$ of men completed secondary school.

\section{Sample and recruitment}

The researcher chose the geographical area based on purposive sampling. Areas selected were based on health data of the women such as the number of women who delivered with TBAs and the distance to public health services. After the areas were selected village midwives, head of the neighborhood and village health volunteers recruited participants based on the inclusion criteria: 1) married women; 2) expecting birth or had experienced birth (TBAs or Midwives); 3) lived in the village and 4) able to communicate in the Indonesia language. Recruitment by the village midwives and village health volunteers (Kader) continued until there were enough participants for 3 groups with 5-6 for each group. The researcher collected data from April to May 2011.

\section{Data analysis}

Data were collected from the focus groups led by the researcher and a trained research assistant using semistructure questionnaires guiding the focus group discussion. Content analysis to determine who said what followed Ezzy's [14] five-step process: (1) Early summaries of the interview were made immediately after transcribing and reading of the interview to obtain a sense of the content; (2) All interviews were coded manually and a large number of categories were outlined and grouped together under higher order headings; (3) Similar headings were removed and categories were generated to reflect the study aims; (4) Trustworthiness of data analysis was addressed by asking a colleague to generate the theme list independently; (5) Every transcript was coded based on the list of themes and then moved to the theme where it belonged. 


\section{Ethical consideration}

The Ethics Review Board in St. Luke's College of Nursing Tokyo, Japan approved the study. The district health office (dinas kesehatan kabupaten) and community health center (puskesmas) in West Java, Indonesia also approved the study. This study was conducted based on the protection of human subject's provisions from the Declaration of Helsinki including anonymity and confidentiality. The objective and significance of this study and the procedures and ethical considerations were explained to the health care staff to gain their cooperation and assistance in this study. After approval of both the ethics committee and district health office the researcher explained the study to the village midwives and village health volunteers (kader). The researcher explained the study emphasizing participant's rights to withdraw at any time without penalty. Informed consent was received from each participant. The collected data will be kept in a safe place for at least three years. After publication, all data will be shredded.

\section{Results}

\section{General characteristic of the women}

As was found throughout Indonesia, most women in this village were Muslim. Of the 16 women interviewed the majority were from Village Dago, West Java. Only six of the 16 women delivered with a midwife and they chose to deliver at home. Yet, all the women went to the paraji for a check-up especially at the fourth and seventh months of pregnancy. In this area the paraji was an important tradition during pregnancy. At four months and seven months of pregnancy, the paraji provided a massage for pregnancy. In essence, the women believed that in order to maintain their infant's health, they needed to take a good care of themselves and follow the cultural rules.

There was a range of issues that influenced women views of the traditional belief during pregnancy. Four global themes emerged: (1) pregnancy is a normal cycle in women's lives; (2) women follow traditional beliefs; (3) follow traditional beliefs relying on paraji rather than midwife and (4) midwives are more secure than paraji.

\section{Pregnancy is a normal cycle in women's life \\ Pregnancy is natural phenomena not sickness}

Most respondents from this village said pregnancy was a normal experience, which should not be regarded as a disease or pathological state. The women also considered pregnancy to be a normal period in their life cycle. They believed that pregnancy would bring them only happiness, even though they did not have any special desire for babies.

One woman said "I think pregnancy is a normal process so you do not need to think bad thoughts about $i t$ ". Another woman mentioned, "Delivery also is a normal process, even though nobody helps you and the baby will come out itself'.

In this case women reported that view regardless of the feasibility that a complication could occur, because they did not think something would happen to them during the process. Therefore danger signs were not neither anticipated or looked for nor even recognized.

\section{Not recognizing danger signs during pregnancy}

Almost all of the women failed to identify the danger signs of pregnancy. Many of the women did not think a headache was a problem during pregnancy. A respondent said: "I think a headache during pregnancy is a normal sign, there is no need to worry about that". Most women had experienced a headache during their pregnancy. One woman said, "I think a headache will come if you eat many foods containing acid like orange or lemon. But there is no danger and it did not cause trouble with their pregnancy". Many of women identified that those symptoms are not necessarily related to pregnancy. They agreed, "There are many causes of headache, if they are late to take breakfast, or are too tired, surely a headache will come. However, a headache is not about your pregnancy, so you just take a rest and eat regularly and it will be going by itself". However, another woman believed when they had some problems, for example headache, they would be better off if they went to the paraji and received a remedy. One woman said, "The paraji gave me Jampe (sprayed water), I feel better after paraji's therapies". She said, "jampe was used for exorcism".

Not all women believed that self-care or care by the paraji was the correct action. On the contrary, a few women said headache is problem during pregnancy and thus sought a check up by midwives, doctor or went to the hospital when they had a headache. A few women agreed, "Headache is not a normal sign, because headache is one of the signs of hypertension. So, we need to get a check-up regularly with midwives". Another woman said, "If headaches are frequent during your pregnancy then that is a great risk and also a risk for your baby". Another woman said," it was a very big problem and I delivered by caesarean operation, so we need to consider going to midwives if we had headache during pregnancy". The prevailing belief however was in the normalcy of pregnancy with whatever risks ameliorated by traditional diet and behaviors.

\section{Infant and mother's death during pregnancy is brought about by God's will}

During the focus group the women mentioned that only a few women and babies died during pregnancy; even if it happens, it is because God wanted it this way. One 
woman said, "A woman was delivered by a paraji and her baby died after delivery. The women said, "Her baby died because of umbilical infection and she knew it was what God wanted and she only surrendered and believed this is fate. So she prays to God to keep us safe. "That is the only thing we will do."

\section{Women follow the traditional beliefs Positive motivation to follow the traditional beliefs}

During the period of pregnancy and childbirth women changed a variety of health-related behaviors including types of food consumed and their activities. There were specific culturally defined prohibitions on eating certain foods during pregnancy and childbirth though not all women followed this cultural rule. Women believed that in order to make themselves healthier they needed to take good of them, as well as follow the traditional beliefs and pray to God to make them safe.

Generally, the women's beliefs about what types of food that should and should not be eaten during pregnancy and childbirth were common within the wider population. A woman said, "Drinking soda (coca cola, sprite) was not good for pregnancy; it will make contractions and your baby will be damaged. Another woman stated "vegetables are better after delivery than meat or fish, because the fish makes the breast milk smell and the taste is bad for the baby and people surrounding them". Women followed the cultural rules of eating to make them selves healthier.

\section{Afraid to not follow the traditional beliefs}

Even though many women had a check-up with a health care provider at the Integrated Health Service Posts (Pos Pelayanan Terpadu (Posyandu), and most of them received information from a midwife about health care during pregnancy and delivery the women said that following the traditions and advice from their grandmother and mother was more important. They followed traditions even though they did not understand why and they believed that if they disobeyed the ritual it would have a negative impact on them. For example "they should avoid going outside at night without jimat (protection such as scissors, garlic, and knife)". Almost all traditional beliefs and practices identified by women were directed toward the consequences of not adhering to the practices. For example one woman said that she went out at nighttime without the jimat, and after that she felt she had a devil in her home. One woman said "I felt uncomfortable if I disobeyed these rituals, this is our tradition, and we will follow all of the traditions even if we could not understand why. It is true. If we did not follow the rituals, after that something will happen to you". Most of women believed that some traditional rituals should be obeyed. They believed all the rituals had a benefit and felt uncomfortable if they did not follow it.

\section{Relying on paraji rather than midwife \\ Parajis are kind, tolerant and patient and have more experience than midwives}

The services of TBA (paraji) were commonly used in Dago Village during the antenatal and childbirth periods. Some women said, in seeking safety and comfort they had a strong belief that the paraji would come help them and would be with them whenever they wanted them. Furthermore paraji's were more patient; they gave them support and were helpful people. One woman said, "I delivered with midwives at home but paraji also was here to help me, and usually after delivery paraji did a kind of massage to restore my body like it was before. Paraji's are also responsible for taking care of the cord and bathing the newborn. Women reported that the paraji lived in their community and they have known the paraji since they were young and had a good relationship. On the contrary midwives were only available during the process and could not help them as much like the paraji did.

\section{More accessible than midwives}

In this area where one village midwife was available, traditional births attendants were more capable of reaching the community. The community also perceived their role to be as important as the Ustadz (religious leader). Some women preferred using the services of the paraji to health care professionals such as the village midwife. One woman said, "I do not need much money to pay the paraji and the paraji did not push us to pay and we will pay when we have money or something to give them." Some said, "I needed to prepare more money to use midwives services, on the other hand using the paraji service we do not need transport because the paraji lives within our community". Regarding transportation they said, "If you delivered at the health centre it was too far away to be reached and also it's hard because you need to use a car or motorcycle". However parajis are part of the community and easily accessible. The common mode of transportation in this village was by motorcycle or walking. In some areas their homesteads were very far away from the regular routes of the commuter motor coach. At night it became difficult to borrow such a car. This was an undesirable situation as it would also be expensive. The women thought money was one barrier for them in seeking health facilities.

\section{Encouragement of natural birth}

Some of women mentioned that the paraji is always besides them during the process, kind and patient and always waiting until the baby comes out naturally. They 
said, "Midwives performed an episiotomy to make the baby comes out faster; sometimes midwives did not have time, so they did another intervention." Only a few of the women had experienced birth at the hospital. They believed birth at home was a normal process. One woman said "I preferred delivery with a midwife; even when delivering with a midwife I did not want to deliver at the hospital or midwife's clinic. It makes me worried. The people in this village would think something bad happened with me. I did not want to make the people thinking badly about me." Thus, they only sought midwives if they had some problem with their delivery.

\section{Midwives more secure rather than paraji Using a medical standard}

However, a few of women preferred using the services of health professionals. One woman said, "The paraji waited a long time until the baby come out; it made me worried whether she could handle the situation. She waited and did not think whether I still had energy or not. But, midwives would give us an intervention to restore our energy through intravenous injection if the delivery was longer than usual." Another woman said, "Midwives give the appropriate care during the process and made us feel safe". One woman said, "The paraji was not so secure; sometime they used a strange method and did not use a glove".

In summary, the practices of parajis were important in this community. Women seemed to favor the traditional belief option for the following reasons; the paraji was easily available, affordable and accessible to the women. The beliefs about health during pregnancy followed traditional beliefs and they followed all relatives' suggestions. They believed God would determine what would happen in their life, so they kept praying and believed that fate was important.

\section{Discussion}

The findings of this study have shown that the ultimate focus of all decisions made by the women who lived in the Village Dago related to traditional beliefs. What they described and what could be observed was a range of different behaviors dependent on their interpretations of concrete life situations. Influencing factors on beliefs was that pregnancy is a normal cycle in women's life, women follow traditional beliefs, women rely on the paraji rather than midwife and a few of women thought midwives were more secure rather than the paraji. The in-depth interviews using focus group discussion have shown that the women in this community had various beliefs about how to manage pregnancy.

\section{Pregnancy is a natural life event}

Women believed that pregnancy was a normal cycle in a woman's life, which did not need special care during the antenatal and childbirth periods; home delivery was more acceptable than traveling to a clinic or hospital. Studies from a variety of cultures in less developed countries have also identified similar beliefs. Similarly, a qualitative study in Bangladesh and Australia [15,16] reported pregnancy identification and its subsequent care was seen as a normal event and was not considered special consideration unless significant complications arose. In addition, an ethnography study by Janson, [17] in Ghana found that some pregnant women in a Ghanaian Village also described childbirth as being something natural and not an illness, which does imply that the pregnant woman can deliver at home.

Additionally some symptoms during pregnancy for example, headache, were considered a normal sign. Agus et al. [5] found that only $62 \%$ of women correctly answered the item: 'headache is a normal sign during pregnancy'. If they needed something for other symptoms including headaches they believed going to the paraji and receiving a remedy would make them feel better. More over, the practice for taking traditional herbal remedies during pregnancy had been passed down for many generations and there were no associated side effects in Indonesia [9].

Although some of these beliefs were culturally understandable, women proceeded on the belief that if they followed the traditional beliefs, practices and advice of family and the paraji then they had done all that they could and the remainder of the outcome was in God's hands. In this village, the women believed that babies die during the process of delivery because of God wanted that way. Belief in God and acceptance of all of God decision was absolute; religion also had an impact on women's perception of how to maintain and preserve their pregnancy. Similarly, a study in Zambia found that illness during pregnancy was explained by culturally accepted causes. They believed a physically and spiritually weak state made one more susceptible to illness, sickness, witches and evil forces in the environment and they accepted it as God's will [18]. Mathole et al. [19] confirmed that Zimbabwe women believed that the first three months of pregnancy were crucial and sensitive; it was believed that a pregnant women and the pregnancy are vulnerable to witchcraft during the early period of pregnancy.

Although pregnancy was considered a normal event in women's live and God is one who gives the decision, following the health care provider information is one part of their effort to preserve their pregnancy. From the medical perspective women can get into trouble during the delivery without her knowledge, because most 
obstetric complications occur around the delivery period and often cannot be predicted. Therefore, they need special attention for their pregnancy to avoid complications, which can be addressed with certain preventive measures by a professional health care provider. More over, the health provider should understand well the women's belief in order to simplify giving the health information.

\section{Women tend to keep and obey the traditional beliefs}

This study found women followed the traditional beliefs. They believed that following the traditional rituals and praying to God would make them become healthier. As a result, one area talked about was what types of food should and should not be eaten during pregnancy and childbirth.

Other cultures have also developed food taboos for pregnant women. Chinese women in Hong Kong found that dietary taboos during pregnancy were: eating shrimp because it will cause skin allergies, rabbit meat, which will cause cleft plates in newborn babies and beef which will also affect fetal health [20]. Fear of not following the traditional beliefs was common in this village; they stated they felt uncomfortable if they disobeyed these rituals. A study in Limpopo South Africa found that to prevent malformation of the fetus, which could be inflicted by jealous people, pregnant women needed to be supported physically and spiritually and also needed be strengthened with herbs [21]. An interesting perspective presented by Camacho, [22] stated that among indigenous women, taboos and permissions during the reproductive cycles were strongly linked to the sacred dimension of nature and spaces were related to the knowledge, practices and rituals.

Empowerment of women is a key strategy in strengthening women's capacities to make healthy decisions for themselves and their families. The empowerment of women is one of outcomes of women centered care (WCC) [23]. Women who had an opportunity to make a decision about choice of care, they felt their views were acknowledged [24]. In addition women who had a strong desire to make decisions were more likely to give birth at a health facility than women with little decision making power [25]. Promoting the exchange of knowledge is a strategy to expand the learning process among women and health care providers. This will help strengthen the effectiveness of the responses of health care providers for the specific needs of women. Empowering women's activities should be woven into health education strategies that promote cultural changes that can benefit women and families.

\section{Who were more affordable health providers the Parajis or midwives?}

We found a main factor determining the type of health care provider for some women in this village was economical.
They selected the paraji because she was more financially feasible. Another reason found was that the paraji was felt to be more kind and patient and more capable of reaching the community. Similarly Titaley's study [11], found the reason for using the services of the paraji were economic, trust, tradition and easy access. In South Africa women agreed that the parajis were knowledgeable in what they did and had skills for pregnancy care [21].

Access between the community and health care center and health care provider also emerged as an issue. For some, home delivery was considered more convenient for some women because they thought they had good health. The home environment was more comfortable than the hospital, and they received support from family members [21]. Women trusted the paraji because they knew them, as they were from the same community and some of their family members were also using the same services during their pregnancy.

Another finding was that a few women in this village stated midwives were more secure rather than the paraji. Wulandari's [9] qualitative study using in-depth interviews in Bali Indonesia found that women stated they only go to midwives and never believed the paraji. On the contrary, women in South Africa stated that they were sometimes reluctant to visit clinics due to the attitude of the nurses [21].

Women who preferred a paraji and those who preferred a midwife both agreed that the paraji was still needed to maintain their pregnancy; they had a strong belief that at the seventh month of their first pregnancy they should go to a paraji to receive a special massage. Therefore, the roles of village midwives and parajis were perceived as vital, particularly in that rural area.

Midwives as health care providers provided comprehensive care during pregnancy. However to increase women's use of midwifery services requires the adoption of some strategies because village midwives were perceived as unacceptable to women and their family. Village midwives need to consider the traditional understandings and beliefs and find a way to be more accepted within the community. One strategy must be to establish a relationship of trust within the community. Trust is an important factor for increasing the use of health provider services. With increasing trust the midwives should be able to conduct more effective health education including important risk factors and actions to take especially if women are primarily using the services of the paraji. Moreover, increasing the parajis knowledge and skill base including appropriate referrals, cultivating a referral system and facilitating the acquisition of health insurance would address part of the motivation to use only the paraji. 


\section{Limitation and further research}

There were several limitations of this study: First, was the small number of women participating in the focus group therefore, the results are not transferable to all Indonesia women. Second was possible bias in subject selection related to interpersonal relationships within the village. Third was the data collection method. Focus groups, while useful for generating rich discussion, have the drawback of not always capturing varieties of opinion because of social conforming especially in some cultural settings. Even so, this study found several noteworthy issues about women's understanding and experiences about pregnancy.

Developing a trusting relationship between midwives and the community is perceived as vital; traditional beliefs should be discussed among midwives, policy makers and planners to increase their understanding as to how to develop strategies for increasing midwives' services. Improving women's knowledge regarding danger signs during pregnancy is a top priority for women. To make them aware about danger signs, improving knowledge is one strategy to avoid complications during the process. A randomized controlled trial study by Liu [26] found that health education for the intervention group was successful in improving pregnant women's health knowledge and some postpartum practices. Future research should focus on describing the beliefs and practices of more women in the area and on the development of strategies for how midwives can improve health education and awareness about women needs. Empowerment of women is one possible strategy for those sets of problems.

\section{Conclusions}

In this study the most dominant provider chosen by women in the village was the paraji. The reasons for this choice were: services of the paraji were kind, inexpensive, interwoven in the community and easy to access. Women believed that because pregnancy was a normal life event, they did not need special consideration during pregnancy. By following their relative's suggestions and trusting in God's will, women thought they would have nothing to fear. These were the important beliefs for maintaining a healthy pregnancy. These were the traditional beliefs. Therefore to be accepted in the community it is vital for the midwife to understand the community beliefs and to develop a trusting relationship between health care provider and community.

\section{Competing interests}

The authors declare that they have no competing interests.

\section{Authors' contributions}

YA and SH were responsible for the study conception and design and the drafting of the manuscript. YA conducted the focus groups and the data collection undertook the data analysis. YA, SH and SP performed the interpretation of the data and editing the paper. All authors read and approved the final manuscript for submission.

\section{Acknowledgements}

I am very thankful to the Health Office District of Vila Dago, West Java for allowing us to conduct this study. I am also indebted to the village midwives of the Public Health Community and Health Volunteers (kader) for helping me to recruit women and establishing the interview schedules. Finally, I would extend a special thanks to all the respondents for their useful information and cooperation. I hope this study will make a positive impact on their lives in the future. This work was supported by St. Luke's College of Nursing, Tokyo. We are also grateful to the school for its generous financial support and scholarly guidance. The Asia-Africa Science Platform Program in JSPS also supported this research.

\section{Author details}

'St. Luke's College of Nursing, 10-1 Akashi-cho Chuo-ku, Tokyo 104-0044, Japan. ${ }^{2}$ Oregon Health \& Science University Portland, Oregon, USA. ${ }^{3}$ Syarif Hidayatullah State Islamic University, School of Medicine and Health Science, Jl. Kertamukti Pisangan Ciputat, Jakarta, Indonesia. ${ }^{4}$ St. Luke's Birth Clinic, 1-24 Akashi- cho, Chuo-ku, Tokyo 104-0044, Japan.

Received: 18 June 2012 Accepted: 24 October 2012

Published: 29 October 2012

\section{References}

1. World Health Organization: Maternal Mortality. http://www.who.int/mediacentre/factsheets/fs348/en/index.html.

2. World Health Statistics: http://www.who.int/whosis/whostat/2008/en/index. html.

3. World Bank: Indonesia Health Sector Review; Accelerating Improvement in Maternal health: Why reform is needed. http://siteresources.worldbank.org/ HEALTHNUTRITIONANDPOPULATION/Resources/281627-1292531888900/ INSPolicyNoteMaternal.pdf.

4. Ministry of Health: Indonesia Reproductive Health Profile. The World Health Organization. http://www.searo.who.int/LinkFiles/Reporductive_Health_ Profile_RHP-Indonesia.pdf.

5. Agus $Y$, Horiuchi S: Factors influencing the use of antenatal care during pregnancy in a rural area of West Sumatra. BMC Pregnancy Childbirth 2012, 12:9. doi:10.1186/1471-2393-12-9.

6. UNFPA: New report shows cultural sensitivity critical to successful development strategies, women's equality. 2008. http://www.unfpa.org/public/lang/en/ News/pid/1375.

7. Leininger M: Becoming aware of types of health practitioners and cultural imposition. J Transcult Nurs 1991, 4:39-40.

8. Aikawa R, Jimba M, Nguen KC, Zhao Y, Binns CW, Lee MK: Why do adult women in Vietnam take iron tablets? BMC Public Health 2006. doi:10.1186/1471-2458-6-144.

9. Wulandari LPL, Whelan K: Beliefs, attitudes and behavior of pregnant women in Bali. Midwifery 2011, 27:867-871.

10. Koblinsky M, Conroy C, Kureshy M, Statnton EL, Jessop S: Issues in programming for safe motherhood. http://pdf.usaid.gov/pdf_docs/PNACK513. pdf.

11. Titaley RC, Hunter LC, Dibley JM, Heywood P: Why do some women prefer traditional birth attendants and home delivery?: A qualitative study on delivery care services in West Java Province, Indonesia. BMC Pregnancy Childbirth 2010. doi:10.1186/1471-2393-10-43.

12. Williams C: Maternal referrals in regionalization of perinatal care study Tanjung Sari Indonesia. http://pdf.usaid.gov/pdf_docs/PDABH552.pdf.

13. Ministry of Health: Indonesian Demographic Health Survey. http://www.measuredhs.com/pubs/pdf/FR218/FR218[27August2010].pdf.

14. Ezzy D: Qualitative Analysis. Practice and Innovation. Crows Nest, NSW: Allen\& Unwin; 2002.

15. Carolan M, Cassar L: Antenatal care perceptions of pregnant African women attending maternity services in Melbourne, Australia. Midwifery 2007. doi:org/10.1016/j.midw.2008.03.005.

16. Choudhury N, Ahmed MS: Maternal care practices among the ultra poor households in rural Bangladesh: A qualitative exploratory study. BMC Pregnancy Childbirth 2011. doi:10.1186/1471-2393-11-15.

17. Jansen I: Decision making in childbirth: The influence of traditional structures in a Ghanaian village. Int Nurs Rev 2006, 53:41-46. 
18. Maimbolwa CM, Yamba B, Diwan V, Arvidson RBA: Cultural childbirth practice and beliefs in Zambia. Issues and Innovations in Nursing Practice. J Adv Nurs 2001, 43:263-274.

19. Mathole T, Lindmark G, Majoko F, Ahlberg BM: A qualitative study of women's perspectives of antenatal care in a rural area of Zimbabwe. Midwifery 2004, 20:122-132.

20. Lee STD, Ngai LSI, Lok HI, Yip KSA, Chung HKT: Antenatal taboos among Chinese women in Hongkong. Midwifery 2009, 25:104-113.

21. Ngomane S, Mulaudzi FM: Indigenous beliefs and practices that influence the delayed attendance of antenatal clinics by women in the Bohlabelo district in Limpopo, South Africa. Midwifery 2012, 28:30-38.

22. Camacho VA, Castro DM, Kaufman R, et al: Cultural aspect related to the health of Andean women in Latin America: Key issue for progress toward the attainment of the Millennium Development goals. Int $J$ Gynaecol Obstet 2006, 94:357-363.

23. Horiuchi S, Kataoka Y, Eto H, Oguro M, Mori T: The applicability of womencentered care: Two case studies of capacity-building for maternal health through international collaboration. Jpn J Nurs Sci 2006, 3:143-150.

24. Tinkler A, Quinney D: Team midwifery: The influence of the midwifewomen relationship on woman relationship on women's experiences and perceptions on maternity care. J Adv Nurs 1998, 28:30-35.

25. Kirrin-Gill MS, Pande R, Malhotra A: Women deliver for development. Lancet 2007, 370:1347-1357.

26. Liu N, Mao L, Sun X, Liu L, Yao P, Chen B: The effect of health and nutrition education intervention on women's postpartum beliefs and practices: a randomized controlled trial. BMC Public Health 2009. doi:10.1186/1471-2458-9-45.

doi:10.1186/1756-0500-5-589

Cite this article as: Agus et al: Rural Indonesia women's traditional beliefs about antenatal care. BMC Research Notes 2012 5:589.

\section{Submit your next manuscript to BioMed Central and take full advantage of:}

- Convenient online submission

- Thorough peer review

- No space constraints or color figure charges

- Immediate publication on acceptance

- Inclusion in PubMed, CAS, Scopus and Google Scholar

- Research which is freely available for redistribution 